Article

\title{
The Logical Dynamics of Information; Deacon's “Incomplete Nature”
}

\author{
Joseph E. Brenner
}

Chemin du Collège, Les Diablerets CH-1865, Switzerland; E-Mail: joe.brenner@bluewin.ch;

Tel.:+41-244-922-118; Fax:+41-244-922-118

Received: 26 June 2012; in revised form: 8 October 2012 / Accepted: 9 November 2012 /

Published: 16 November 2012

\begin{abstract}
In his Incomplete Nature, Deacon extends a thermodynamic concept of energy to yield a description of complex processes in which absence plays a critical role in their emergence and evolution. Starting from a quantum-mechanical picture of energy as an energy-matter duality, the critical role of potential as well as actual properties of processes is also described in the new extension of logic to real phenomena, Logic in Reality (LIR), which I have proposed. Deacon shows how an interactive operation of both Shannon entropy and Boltzmann entropy must be taken into account in information. Here, I demonstrate the complementarity of our two approaches to what is not, or not fully, present for an understanding of the dynamics of complex phenomena, especially, of intentionality, information and meaning. Deacon shows that the hallmark of information is its absent content, and LIR shows that presence (actuality) and absence (potentiality) in such processes are related dynamically. Deacon's approach and LIR ground and extend Logan's concepts of biotic information and the relativity of information vs. meaning. Their conjunction constitutes a new conceptual structure for exploring the relationship of information to materiality, that is, to the matter-energy that constitutes it as its carrier and/or substrate.
\end{abstract}

Keywords: absence; actual(ity); constraint; contradiction; dynamics; emergence; logic; meaning; opposition; potential (ity); process 


\section{Introduction}

\subsection{The "Invisible Hand" of Information}

In 2004, seeking a new understanding of informational phenomena based on the molecular organization of life [1] Pedro Marijuan stated flatly that "rather than resorting to the special, and rather vacuous, terms of autopoiesis, autogenesis, self-production, etc., one can simply say that living existence is informational. Understanding the 'invisible hand' of information should not rest exclusively on a vast catalog of computational processes and models addressing physico-molecular occurrences, but also on making integrated sense of the differentiated ways of existence that a living system enacts. The invisible hand of information is the great shaper of the natural and social world. Continuous communication between self-constructing agents leads to an endless variation in their constitutive structures, involving both production and degradation processes which stem out from a generative blueprint or from any other form of a slowly changing 'identity'.”

Since this paper appeared, there have been many attempts to pin down the nature of the "invisible hand", as well as the form of the communication between agents, but this has proven extremely difficult. Mathematical approaches, such as that proposed by Marijuan himself of the partitional canon, have not led to many fundamental new insights. It is becoming clearer today, however, that no single explanation derived from any single classical discipline could be adequate, as is shown by recent studies by Hofkirchner [2], Burgin [3], and Luhn [4] as well as Marijuan and myself.

Two new groups of studies, which have appeared only in the last two or three years, are in my opinion significant breakthroughs in the comprehension of the science and metaphysics of information:

- $\quad$ The dynamic absential approach of Terrence Deacon [5,6];

- The informational ontology of Wu Kun, developed over the last thirty years, but which has just begun to be available in English [7].

As I will show, my own new approach involving a non-standard logic (Logic in Reality, LIR [8]) both supports and is supported by this work. LIR is a new kind of logic of the evolutionary development of the real physical processes that underlie existence, grounded in the fundamental quantum self-dualities and dualities of our universe.

As Wu Kun states, it is rather disconcerting to note that in order to approach correctly a concept that is considered part of standard science-information-we must in fact reexamine the entire philosophical structure of transmitted human knowledge. That few people are willing or able to do this is perhaps unfortunate, but it does not change the situation. People who seek certainty and complete definitions for complex phenomena must face continued disappointment. The recent paper by Edouard Machery, "Why I stopped worrying about the definition of life... and why you should as well" [9] should be read by everyone working in information.

The multi-faceted scope that Robert K. Logan has defined for this Special Issue clearly reflects the complexity of the problem and the necessity of seeking "new windows" as has Robert E. Ulanowicz in his closely related analysis of a new paradigm for natural processes [10].

Since Marijuan's paper appeared, there have been many attempts to pin down the nature of the "invisible hand", as well as the form of the communication between agents, and even the definition of 
what constitutes an "agent” has proven extremely difficult. One problem, suggested by a reviewer, is that that there is no exact unambiguous definition of an agent. Traditional definitions assumes that an agent is a system who/that acts on behalf of or/and represents another system or other systems. A more modern approach treats an agent either as a system who/that exerts some force or effect or as a system who/that acts or has the power or authority to act. Such a definition covers both the internal structures suggested by Marijuan, and human creators and senders of information and is satisfactory for my purposes here. According to the traditional definition, any agent is an operator because to accomplish his/her/its mission, the agent has to operate. However, there are operators that are not agents because they are aimed solely at their own goals, as discussed by Burgin in his general theory of operators. Much of the literature about agents, in addition, deals with the existence of so-called free agents as the basis for free will. Further discussion of the concepts of agents as such is, accordingly, outside the scope of this paper.

\subsection{Rationale}

There is general agreement that information is a complex but perhaps unifying concept that nevertheless comes in a wide variety of forms. However, the current set of assumptions about its nature, still based largely on computational extensions of Shannon's original ideas, is sufficient to explicate its minimal physical characteristics but insufficient to define its representational character or its functional, qualitative and normative value.

This paper discusses the new approach to information, proposed by Terrence Deacon, as a process instantiating a complex dynamics that starts with thermodynamics and continues throughout higher ontological levels of form (morphodynamics) and intentionality (teleodynamics). In his Incomplete Nature (hereinafter $\mathbf{I N}$ ), Deacon extends a thermodynamic concept of energy derived from statistical mechanics to yield a complete and cohesive description of complex processes, in which absence plays a critical role in the emergence of living systems, mind and information. Starting from a quantum-mechanical picture of energy as an energy-matter duality, the critical role of potential as well as actual properties of processes is also described in the new extension of logic to real phenomena, Logic in Reality (LIR) which I have proposed. LIR argues that the fundamental physical structures of the world instantiate a logic as well as a mathematics. In fine, Deacon shows how an interactive operation of both Shannon entropy and Boltzmann entropy must be taken into account in information, and Logic in Reality (LIR) further suggests that information involves non-Markovian processes.

In this paper, I demonstrate the complementarity of the LIR and Deacon approaches to what is not, or not fully, present-not "there"-or an understanding of the dynamics of complex phenomena, especially, of intentionality, information and ethics. Deacon shows that the hallmark of information is its absent content, a resultant function of the necessary physicality of information processes, and LIR shows that presence (actuality) and absence (potentiality) in such processes must be related dynamically. While the importance of a concept of absence for information was indicated by Marijuan ten years ago, it is Deacon's detailed current development that now calls for our attention.

Logic in Reality (LIR) is a new kind of non-propositional logic that extends its domain to real phenomena. Its axioms and rules provide a framework for analyzing and making inferences about complex real world entities and interactive processes at thermodynamic, biological and cognitive-social 
levels of reality or complexity. Due to its own rigorous ascent from the properties of matter-energy, it provides a reconciliation of the logic of physical science and the logic of living and mental teleology and can link energy, form and information. It offers a new approach to the nature of physical causality and a way to use potentialities to achieve teleological properties from unambiguously non-teleological starting points. Despite the prestige of Norbert Wiener and John Wheeler, it is becoming clear that their-related - statements to the effect that energy is not information and information is primitive to matter — energy (it from bit) have been profoundly misleading.

In terms of this special issue, the relation of modes of information to meaning, Deacon's approach and Logic in Reality (LIR) accomplish several objectives: first of all, they ground and extend Logan's concept of the relativity of information, in that information is not only not an invariant quantity, but a process or set of processes of processes. As we will see, Deacon's description of the evolution of intertwined thermo-, morpho- and teleodynamic levels further develops the concept of biotic or instructional information that results in the propagation of organization as pointed out by Kauffmann, Logan and their colleagues [11]. In their concept of biotic information, biological or cognitive meaning is defined by interaction with the context (or environment) that interprets the information.

LIR provides a necessary further validation of the role of absence as defined by Deacon, in relation to presence, and prepares its valuation. Valuation of absence and negation in general is logically equivalent to the valuation of the other, immanently. Thus in addition to explicating the evolution of complex processes, Logic in Reality, unlike all standard logics, founds an ethics and this implies, today, an ethics of information. It supports the call Deacon makes for rethinking the framework of the natural sciences in a way sophisticated enough to integrate absential or potential phenomena. The conjunction of Logic in Reality and Deacon's dynamics constitutes a new conceptual structure for exploring the relationship of information to materiality, that is, to the matter-energy that constitutes it as its carrier and substrate.

A vision of reality that includes subjectivity and moral value, without requiring any anti-scientific principle to justify individual and collective moral responsibility, can be defended. Deacon's view of the dynamics involved in informational processes supports a new and central role for information in the reform and naturalization of philosophy itself.

In this paper, I will show that the work of Deacon and Logic in Reality share the ontological feature of being firmly grounded in physical processual reality. I will now show that the latter enables a critique of the former such that principles of the combined theories offer a new platform for progress in the science and philosophy of life.

\subsection{Overview of Paper}

I begin in Section 2 by listing some of the philosophical, logical and phenomenological problems associated with current definitions of information. This has the concrete goal, consistent with the objectives of this Journal, of opening the debate to perspectives from a number of new disciplinary sources.

Section 3 is a summary of the Deacon theory of incompleteness and the critical role of absence in nature. In my opinion, when a very new conception appears, despite or perhaps because of the fact that 
it challenges much received wisdom, it is essential both to acknowledge it existence and see "how far it takes one". I have tried to do this here.

Section 4 is essentially a summary, similar to what I presented in a previous paper in this Journal, of the principal tenets of Logic in Reality (LIR). As usual, I have had to strike a balance between presentation of the complete theory and what is necessary for this analysis. I had the objective of using LIR in a self-referential manner. In Sections 3 and 4, the reader will first see how the theories are "the same and different” (an LIR concept) in their treatment of energy.

Section 5 is a transitional section that discusses in general terms the inversion of perspective (figure-background shift) that is a key feature of both Deacon's theory and LIR. The goal here is to make sure that some of the essential insights that go back to Gestalt theory are not forgotten in relation to current issues.

Section 6 presents some of the key philosophical and physical issues and dualities that have received treatment by both Deacon and me, bringing out their agreements and disagreements and their consequences for information. It is a consequence of Logic in Reality that it forces one to reconsider the basis for almost every critical concept in science and philosophy as they apply to the theory and science of information: complementarity, circularity, emergence and so on as well as absence.

Section 7 is my central discussion of information and meaning. It is the briefest possible exposition of the application of the principles of LIR to the complex of processes for which the shorthand is "information".

I conclude in Section 8 with a discussion of the new informational paradigm emerging in the work of $\mathrm{Wu}$ and Capurro as well as Deacon, Logan and others including myself. My goal is to give the maximum possible substance to the term "information paradigm" for others to build on.

\section{Problems in Theories of Information}

Deacon emphasizes that his theory and its conclusions are independent of what the ultimate underlying structure of the universe may be. However, the picture that Logic in Reality gives of that structure is compatible with most of his conclusions regarding the evolutionary dynamics of emergent phenomena. It is possible to imagine that both approaches include significant insights and may be combined to give a more complete picture of reality than either by itself. It is the objective of this paper to demonstrate this.

The protagonists in this colloquy are thus Professor Terrence W. Deacon, Dean of the Department of Anthropology at the University of California, Berkeley and the Franco-Romanian thinker Stéphane Lupasco (Bucharest, 1900-Paris, 1988), the latter “represented" by myself. For the understanding of the synthesis of the views that I am attempting, it is important to understand that Deacon appears to be a monist at the fundamental level, that is, in his picture of the role of spontaneity and the properties of energy, but not at higher biological and cognitive levels of reality, at which his conception of the interactions at and between levels approaches and is supported by that of Lupasco.

From a formal standpoint, I will be addressing the following issues:

(i) The fundamental origin of phenomena and their characteristics;

(ii) The form of the interactions taking place during the evolution of those phenomena;

(iii) The recursive origin of the phenomena at higher levels in the interactions themselves. 
Unlike most theories of this kind, including mine, the description of Deacon has the requisite variety (in the Ashby sense) involving the interaction of energetic, dynamical processes at thermodynamic, morphological and teleological levels [6], roughly corresponding to the levels of reality of LIR [12]. For both theories, it is the interweaving of these processes that can result in the emergence of intentionality and, ultimately, of human self-awareness, conscious interpretation and meaning. By going to one or two additional lower levels of quantum physical reality, however, LIR provides a further ontological basis for the capacity of processes to interact, emerge and interweave.

\subsection{The Problem of Phenomenology}

This paper starts from the assumption that there are clear scientific, logical and philosophical reasons for the difficulty in prior attempts to formulate a coherent theory of the nature and function of information. These reasons can be summarized as a fundamental unidimensionality and lack of complexity in the philosophical concepts and underlying dynamics attributed to informational processes. In the conception of $\mathrm{Wu}$ Kun, much current work in the field of information still assumes a Husserlian limitation of its basis to an internal world, with the bracketing or suspension of the objective world of the subject's own existence.

This position is echoed in the very important book of Sören Brier, Cybersemiotics [13]. Brier challenges us to find “...a new broader and deeper foundation... a reflective metaphysical frame..." for information than theories of dissipative structures, autopoiësis, and Husserl's phenomenology as it appears in Luhmann's reductionist theory of meaning. "We cannot simply add information theory on top of the old mechanistic foundation, or use a physicalist foundation improved by complexity theory or random dynamics as none of these theories includes a theory of the emergence of life, meaning and language.” It should be understood, thus, that the subtitle of Brier's book: Why Information is not Enough, refers only to standard computational notions of information, ultimately tied back to a Peircean ontology whose physical foundations (spontaneity, continuity) are open to question. Nevertheless, Brier would like to retain as much as possible of Peirce's insights compatible with an interpretation in which living systems are the universe's way of becoming aware of itself. The positions developed in this paper, which point toward a new view of the basic problems of the philosophy of information in particular and the fundamental shift taking place in it, are all attempts to give a logical and scientific grounding of this statement.

\subsection{The Problem of Logic}

As I have discussed elsewhere, no satisfactory formal or informal logic has been available for information. Standard truth-functional logics can address the formal mathematical aspects of computational theories of information but not more. Other references to a "logic of information" are simply metaphorical restatements of some of its regularities. Floridi’s Information Logic (IL), or Logic of Being Informed [14], recognizes something static and abstract about standard formulations of doxastic logics that depend on the (tripartite) notion of knowledge as justified true belief. As I will show, however, Logic in Reality ascribes a logical, non-metaphorical content to descriptions of an antagonistic interaction between the individual and the world, as an on-going process. It refers to an informational process in which both actors change as the reactions of one or the other alternately 
predominate, following the precept of Zhong [15] that information cannot be separated from the human actors involved.

\subsection{Avoiding "The Ghost and the Machine"}

The title of this Section refers to two kinds of argumentation and analysis that Deacon goes to some lengths to show must be avoided in discussing complex natural phenomena in general and information in particular. One is what he calls "homunculus" arguments, where homunculus is an emblem for any abstract principle in a scientific or philosophical explanation that imports an unanalyzed attribution of information, sentience, reference, meaning, purpose, design, self, subjective experience, value, and so on-attributes often associated with mental states-into scientific explanations. The origin of this attitude can be traced back to Cartesian mind-body dualism, deconstructed in modern times by Gilbert Ryle, who coined the phrase "The Ghost in the Machine”.

Deacon takes the golem as the avatar of the opposite of a homunculus: apparently mind-like processes that are nonetheless devoid of any intentionality or ententional properties of their own. Ententional is a generic adjective that goes beyond simple directionality. It is proposed by Deacon to describe all phenomena that are intrinsically incomplete in the sense of being in relationship to, constituted by, or organized to achieve something non-intrinsic. If a homunculus is a little man in my head, then the golem is a hollow-headed man, a zombie. The golems in our society are computers and those who believe that everything can be accomplished by them.

The "existence" of golems might be seen as the consequence of investing logic as usually understood with animate power. One problem lies in the usual assumption that syntax and semantics, logic and representation, are independent of one another. A golem is syntax without semantics and logic without representation. There is no one at home in the golem because there is no internal representation possible-no meaning, no significance, no value, just physical mechanism, one thing after another with inflexible consistency. Golems are idealized metaphors. Formal logic assumes that the variables of its expressions are idealized representations, and it brackets the underlying realities from consideration to explore the purely formal, inert relations that must follow from its application.

It is possible, however, to conceive of and develop a logical system, a way of looking things logically that is not a golem, but a bona fide part of natural science, available for other types of inference without re-introducing homunculi. As a "guarantee” of LIR's difference in kind, I can say already that it can accept and handle inconsistency, the inevitable accompaniment to the real world. The reader will have the opportunity of judging, from Section 4 below, whether Logic in Reality accomplishes this objective.

\section{Deacon's “Absential” Theory of Incomplete Nature}

Two transitions have been required for this paper to have come into existence: the transition from non-living to living systems, and the transition of living systems to those capable of thought. Despite millennia of application of thought to its own existence, there is still no minimally rigorous account of what is required to cross these thresholds. This is what Deacon has found new ways of doing which it is my intention to both support and critique. 


\subsection{Absence}

Information, like other complex biological, cognitive and social processes are all, in Deacon's term ententional, that is, they display some form of purpose. Ententional is a generic adjective proposed to describe all phenomena that are intrinsically incomplete in the sense of being in relationship to, constituted by, or organized to achieve something non-intrinsic. There is room for meaning, purpose, and value in the fabric of physical explanations, because these phenomena effectively occupy the absences that differentiate and interrelate the world that is physically present.

They are teleological phenomena, evolving in teleological processes. (The term must not be confused with that coined by Collier [16] of enformation. Enformation is part of a system's invariant structural information, which is required for causal connections in it to exist from time $t_{0}$ to $t_{1}$. The concept is intended to emphasize that chance regularities cannot carry information that describes the internal aspects of such phenomena.) Ententional processes are notoriously recalcitrant to study by the rigorous methods of the experimental sciences. However, yet if it is agreed that they do not take place outside the laws of physics and chemistry, what is or are the still unrecognized characteristics of the precursors to those processes that enabled them to interact in such a way as to insure the transition from non-living substrates to primitive living systems, and from these to the complexity of human cognition and society?

Deacon deserves the historical credit for showing that that general characteristic is a negative relationship defined with respect to absence. The concepts of information, function, purpose, meaning, intention, significance, consciousness, change and human value are intrinsically defined by their fundamental incompleteness [5,6].

Very briefly, Deacon's argument is that the existence of anything requires the imposition of some force that separates it from other things-a constraint; this constraint reduces the number of possible states of the entity which is, accordingly, defined by what it is not, or what it is not yet. The absence (sic!) of any serious literature about absence as a physical and metaphysical principle that is causally efficient is, negative, evidence of the resistance to or lack of interest in it. Only in some theories of deontic (judicial) logic is negative evidence given appropriate attention. Apophatic philosophy is about the importance of things that are not said, but not about things that are not there.

In order to demonstrate the importance of reference to physically absent or absential aspects of phenomena as critical to their understanding, it is necessary to begin with the simplest things that happen at the lowest relevant level of physical reality. At this first stage in our discussion, this will be the level of macroscopic physical phenomena at which the laws of classical physics and thermodynamics, available since the 19th Century, hold without violation.

\subsection{Absence and Constraint. Levels of Dynamics}

The 2nd Law of Thermodynamics describes the energy gradient in which the world has existed since the Big Bang (or its equivalent in a cyclic model of the universe). The existence in this gradient of existence of spontaneity and spontaneous processes corresponds to the familiar phenomena of "water running downhill” or of chemical reactions taking place if the free energy of the products is 
lower than that of the reactants. In other words, spontaneous processes are those described by Newton's 1st Law of Inertia.

The concept of constraint can provide a negative "absential” approach to real phenomena that are determined by discrete interaction effects that do not occur spontaneously. Constraint is in effect, a complementary concept to order, habit, and organization, because it determines a similarity class by exclusion. However, it is essential to remember that a constraint is not in and of itself a totally negative property or entity. As indicated below, nothing causally effective can be defined by total absence or negation, and in this LIR differs from Deacon. Paying attention to the critical role played by constraints in the determination of causal processes is an example of a figure/background reversal (see Section 5). That is critical in developing a scientific theory of emergence, evolution and information. Deacon avoids assuming that abstract properties have physical potency, and yet does not abandon the notion that certain general properties can produce other general properties as causal consequences. This is because the concept of constraint does not treat organization as though it is something added to a process or an ensemble of elements. It is not something over and above these constituents and their relationships to one another. And yet it neither demotes organization to mere descriptive status nor does it confuse organization with the specifics of the components and their particular singular relationships to one another. Constraints are both (1) what is not there but could have been, irrespective of whether this is registered by any act of observation, and (2) what is really there and is the proximal cause of an absence.

In statistical mechanics, constraint is a technical term used to describe a reduction of degrees of freedom of change or a restriction on the variation of properties that is less than what is possible. In colloquial terms, constraint tends to be understood as an external limitation, reflecting some extrinsically imposed factor that reduces possibilities or options. In each case, there are reduced degrees of freedom that in the absence of these constraints could potentially be realized. It is the causal efficiency of constraints as operators [17] that enable the interaction of processes at the different levels of dynamical organization proposed. These levels are:

- Homeodynamics; operation of thermodynamics as noted; characterized by: increase in entropy; dissipation of constraints; tendency toward static equilibrium.

- Morphodynamics; operation of self-organization; characterized by: amplification of constraints; dynamic equilibrium; meta-stability.

- Teleodynamics; operation of living systems; recursive self-reconstitution and reproduction of systems of constraints.

In order to understand how these dynamics have the outcomes they have, Deacon introduces the further notions of orthograde and contragrade processes.

\subsection{Orthograde and Contragrade Processes}

Orthograde processes is Deacon's term for changes in the state of a system that are consistent with the spontaneous, "natural" tendency to change without external interference. The change of a thermodynamic system toward a state of increased entropy, if totally unperturbed by extrinsic influences, is the most basic example of orthograde change. In the real world, of course, nothing is 
ever completely isolated, and no physical system remains forever unperturbed by external influences. This means that thermodynamic orthograde change is often resisted, modulated, or reversed, depending on the relative degree and duration of interaction with other contrary constraints or processes. (I have some questions about the use of “often” rather than "always” here, but let us not interrupt Deacon's train of thought). In any context, orthograde processes will continue until they reach a state in which there is symmetry in the probable directions of change, or until the supportive conditions change. This dynamical terminus of an orthograde process is its attractor, which may or may not be a quiescent state.

Contragrade processes are changes that must be extrinsically forced because they run counter to orthograde tendencies. The assumption of spontaneity is fraught with problems, but I will retain the term for the time being. It remains true that the apparent "spontaneous" tendency of molecules with the potential for interaction to wander into each other's spatial positions and dynamical values is responsible for the orthograde dynamic that characterizes global change toward equilibrium. In this way, contragrade dynamics at one level produce orthograde dynamics at the higher level. Another merit of describing change in these complementary terms is that it gives new meaning to the defining property of matter - a resistance to change - and the defining property of energy - that which is required to overcome resistance to change. Since orthograde processes ensue spontaneously, they are ubiquitously present, even during processes of contragrade (forced) changes. A contragrade change must therefore derive from two or more orthograde processes, each in some way opposing the other's effects. Each must constrain the other in a reciprocal way. So, to again describe this in the negative, the evolution of increasingly complex forms of constraints has given rise to increasingly varied ways to impose constraints on the world with respect to these internal constraints. In this sense, the source of agency can be described as the generation of interactive constraints which do work to perpetuate the reciprocal maintenance of the constraints that maintain an organism or a self.

\subsection{Morphodynamic Processes}

Morphodynamic organization emerges due to the interaction of opposed thermodynamic processes (e.g., perturbation and equilibration), and it results in constraint amplification rather than constraint dissipation (i.e., increase in entropy). In this respect, formation of primitive systems capable of self-replication, described by Deacon in a thought experiment as "autogens", exemplifies the defining feature of an emergent phase transition - the appearance of a new form of orthograde organization. Morphodynamic regularity establishes a new level of dynamical covariance between the rate of entropy increase and the rate of work countering this tendency. This is why self-organized regularity of a low order of complexity is a special case, not the rule in nature, as we recognize from the repeated use of certain examples.

Morphodynamic processes develop in persistent far-from-equilibrium conditions because the increase in internally generated dynamical constraints is more efficient (requires less work) to deplete the energetic and/or material gradient that is driving the system away from equilibrium. In this way, morphodynamic processes accelerate the destruction of whatever gradient is responsible for generating them. They are, in this respect, self-undermining, and are only maintained when this gradient is constantly replenished by some extrinsic source. 
Analogously teleodynamic organization emerges due to reciprocally organized morphodynamic processes, and results in constraint stabilization rather than constraint amplification, and entropy ratcheting rather than entropy production.

As one reviewer has pointed out, these concepts instantiate a property of "asymmetry" in time that is antagonistic to symmetrical classical laws of physics. As György Darvas has shown, much in the spirit of LIR, symmetry and anti- or asymmetry stand in a dialectical relationship.

\subsection{Teleodynamic Processes}

In Deacon's terms, teleodynamics refers to a form of dynamical organization of real processes that exhibit purpose or end-directedness and consequence-oriented features that is constituted by the co-creation, complementary constraint and reciprocal synergy of two or more strongly coupled morphodynamic processes. It results in constraint stabilization rather than constraint amplification, and entropy "ratcheting” rather than entropy production.

In somewhat different terms, in teleodynamic organization, Deacon argues, one sees for the first time that one physical system is capable of influencing other physical systems via something that is merely virtual-that which is specifically absent, missing, displaced, potential, or merely abstract. In the simple thought experiment (antigens) that exemplifies the core architecture of this argument, it is the premature halting of the component morphodynamic processes, a tendency that is spontaneous and would otherwise run to self-extinction, that makes self, information, and life possible. It is the possibility of briefly building up constraints by morphodynamic action, and then halting the process before there is any dissipation of those constraints, but enabling their subsequent propagation, that is the secret of this distinctive form of causality.

When so presented, however, this argument begs the question of the locus of the capacity to so influence other systems, and it is here that Logic in Reality, that it its ascription of the Principle of Dynamic Opposition (see below, Section 4.2), also at a sub-molecular level, that provides the physical basis for the subsequent development. Nevertheless, being able to trace in detail each step that is required to cross from the realm of simple mechanical processes into the realm of ententional relationships provides a new level of explanation, as we will see later in this paper. Even such basic concepts as work and information take on new meaning, and the notions of self and sentience can be given a more precise physical formulation.

In more detail, Deacon's definition of teleodynamics is: a form of dynamical organization exhibiting end-directedness and consequence-organized features that is constituted by the co-creation, complementary constraint, and reciprocal synergy of two or more strongly coupled morphodynamic processes. Thus, while living systems do indeed incorporate morphodynamic processes at nearly every level of their organization, they also exhibit properties that differ radically from those characterizing morphodynamic processes, inverting their "logic". The most important inversion is that. Organisms actively reorganize their internal dynamics and relationships to the environment in ways that specifically counter or compensate for any depletion of the gradients that is necessary to maintain their dynamical integrity and their capacity to so respond.

The ways that these processes all serve to maintain the capacity for self-repair and self-replication exemplify an inversion of the most typical features of morphodynamic systems. This indicates that 
dynamical systems approaches limited to morphodynamic and chaotic non-linear dynamics are insufficient to account for living dynamics, and I have made a similar critique of dynamic systems theory in [8]. Life's paradoxical dependence on morphodynamics, but its inversion of its most characteristic consequences, suggests that an additional dynamical inflection separates living processes from morphodynamic processes. This phase change thus exemplifies a further emergent transition Described in these general terms, one can begin to define this higher-order dynamical "logic" as a dynamical form of organization that promotes its own persistence and maintenance by modifying this dynamics to more effectively utilize supportive extrinsic conditions.

A good example of how the three dynamic levels are tied together in Deacon's theory is in what he calls the self-assembly of macromolecular structures as a special case of crystallization. "Like crystal lattice formation, the growth of multi-unit macromolecular structures is an expression of the intrinsic geometry of component molecules, the collective symmetries these offer in aggregate, and the lower energy state of the crystallized forms. It is in this sense an expression of a thermodynamic orthograde tendency to reduce total entropy. It's just that molecular shape and charge features, and their relationships to other molecules_-including especially to the surrounding water-are responsible for the spontaneity of their growth. The accretion of molecules into crystalline like arrangements is thus a function of thermodynamics, but the influence of their structural and charge characteristics may contribute to the amplification of constraints, thereby generating regularities in the ways they form into aggregates; a morphodynamic consequence.”

From the LIR standpoint this concept is not incorrect, but it is incomplete to base the ascent solely on the intrinsic (actualized) geometry of the substrate molecules, ignoring the role of the potentialized, hence partially absent, energies of those molecules. Self-assembly, like self-organization, are concepts in which "self-" should perhaps be starred_- "self*_"-to remind the reader of the more fundamental dynamics involved.

\subsection{Work}

Deacon's explanation of the way in which the linked thermodynamic, morphodynamic and teleodynamic processes operate is based on work. It is work, rather than energy per se (as in LIR to date) that describes the capacity of the different process components, at and between their respective levels, to lead to the emergence of complex cognitive phenomena. What has been missing is a full understanding of how the power to effect change at all levels is interconnected and interdependent, and Deacon believes an expansion and generalization of the concept of work is the critical first step.

Work is defined in physics as the scalar product of a force times the distance through which it acts. The 1st Law of Thermodynamics states that when work is done to a system, the system's energy changes by that amount of work, equating work and energy. As Deacon points out, however, the concept of work is primitive in the sense of being reflected in the extent of the non-spontaneous change that actually takes place, whereas another statement of the 1st Law refers merely to the fact that there is no change in the total amount of energy of the total system. Work can be defined generally as the production of contragrade change at all three major dynamic levels: at each level there is a class of orthograde and contragrade tendencies in which contragrade change can only be effected by pitting orthograde processes against each other. 
The advantage of couching the discussion of cognitive processes in terms of work is that it permits more intuitive ways of seeing the links between mental work and the physical forms of energy also involved. A general theory of work should demonstrate the isomorphism between physical and mental activities, the ways in which that both energy and ideas can introduce non-spontaneous change into the world.

The concept of work as the common feature in attributions of causal power untangles the notion of causal power from undifferentiated notions of causality. Whereas commonsense conceptions of cause tend to be applied to all kinds of physical changes, attributions of causal power are typically invoked in a more restricted sense. For Deacon, this distinction parallels the distinction between changes due to work and changes in general. Power is an indication that work is involved and not just causality in general-in other words, it invokes a sense of overcoming resistance, of forcing things to change in ways that they would not otherwise change.

Deacon sees this view of causality as a start on formalizing the relationships that link energetic processes, form generation processes, and social-cognitive processes. It shows that what emerges in new levels of dynamics is not any new fundamental law of physics or any singularity in the causal connectedness of physical phenomena, but rather the possibility of new forms of work, and thus new ways to achieve what would not be able to occur spontaneously. Possibilities of new forms of work are made possible by heterogeneous environments, whereas classical laws deal only with homogeneous ones. It is this feature that makes Kauffmann's “adjacent possible” combinatorially intractable and/or non-computable. As I have proposed elsewhere, LIR, which defines the dialectics linking homogeneity and heterogeneity, is the logic of non-computability. The possibilities of generating increasingly diverse forms of non-spontaneous dynamics can produce causal relationships that radically diverge from simple physical and chemical expectations, and yet still have these processes as their ground.

Teleodynamic work can be defined as the production of contragrade teleodynamic processes. Since this must be understood in terms of orthograde teleodynamic processes, the first step in describing this level of work is to define and identify examples of orthograde teleodynamics. In general terms, an orthograde teleodynamic process is an end-directed process, and more specifically, one that will tend to occur spontaneously. Although teleodynamic processes are incredibly complex, and an explanation of the structure of teleodynamic work is by far the most elaborate-since it is constituted by special relationships between forms of morphodynamic work-it is also the most familiar, since it includes the highest level of human cognitive processes.

It should be obvious that Deacon's schemes are, like all others, dependent on prior work not all of which can be adequately referenced. Parallels can be found to Alice Juarerro's dynamics of action and to Robert Rosen's work on anticipatory systems, where the phenomenon anticipated is by definition “absent”. In private communication, Deacon has indicated his intention of rectifying these omissions.

\section{Logic in Reality (LIR)}

The nexus of the LIR and Deacon approaches can be found in Deacon's statement that the causal logic of spontaneous physical processes is radically different from the ententional logic of life and mind. For me, the first logic is standard bivalent logic, adequate for macrophysical phenomena and the 
second is Logic in Reality (LIR), that of dynamic opposition, non-separability and emergence. I therefore turn to the necessary summary of LIR.

\subsection{Opposition and Diversity. The Pauli Exclusion Principle}

I begin my development by describing the consequences of operation of the 2nd Law of Thermodynamics in our world as a spontaneous increase in entropy, that is, disorder. Energy moves from diverse, heterogeneous high-level forms toward a single, homogeneous low-level form (heat). Let us designate such a tendency toward a single undifferentiated state of affairs as exemplifying identity in an ontological sense or, simply, identifying or "homogenizing" (an "identification") leading ultimately to the disappearance of all structure in a smoothed-out homogeneous identity, the "heat death" of the universe. As Deacon points out (cf. Section 3.6) what is important in this picture is the change in the capacity of energy to do work.

Nevertheless, the 2nd Law can be "abrogated" locally and can give rise under certain conditions to the emergence of order and new not-previously existing structures. What has not been generally recognized, however, is that another principle exists in the basic structure of matter that enables new order to emerge and can thus be considered as the opposite of the 2nd Law although operating in a different way, namely, at the level of atomic particles themselves rather than in their statistical behavior.

This second most fundamental aspect of energy is expressed in LIR as a fundamental duality of otherwise indistinguishable electrons, or more generally fermions shows a fundamental duality governed by the Pauli Principle of Exclusion. Electrons are located in shells around the nucleus of an atom, but two electrons in the same shell cannot have the same quantum numbers for their properties, such as spin. Build-up of a multiplicity of shells is possible, for atoms heavier than helium, in which the electrons will all have, as a consequence of their distance from the nucleus and the degree of completion of the shells, a different capacity (potential) for reacting with other atoms to form different molecules enabling the existence of, ultimately, life and human beings. The difference between spin-up and spin-down electrons is thus the earliest example of an internal "difference". The differences between fermions and bosons themselves, the external difference between odd and even spin, or between different kinds of particles, hadrons and leptons are external. A similar phenomenon, due to Pauli exclusion at the nuclear level, results in the existence of nuclear spin isomers. The diversity is real and leads to differences in some physical properties as in, for example, molecular ortho- and para-hydrogen, which have been separated and characterized. However, the effect on chemical properties is negligible.

Energy and accordingly all existence thus also show a tendency toward or instantiate an opposing process of heterogeneity, or non-identity or diversity, a 'heterogenizing' process, a diversification, opposed to the trend toward identity expressed in the 2nd Law. Logic in Reality is the system of thought that sees the existence of opposition as a logical principle, the basis for another description of orthograde and contragrade processes in logical terms. 


\subsection{LIR. Axioms and Calculus}

The most important feature of Logic in Reality (LIR) is that it is logic of relations, especially, of changing relations. LIR is thus a new kind of non-propositional logic that extends its domain to real phenomena, and allows patterns of inference to be made about the evolution of those phenomena. It is based on the work of Stéphane Lupasco [18]. LIR is grounded in a particle/field view of the universe, and its axioms and rules provide a framework for analyzing and making inferences about complex real world entities and interactive processes at thermodynamic, biological and cognitive-social levels of reality or complexity.

The term Logic in Reality implies both (1) that the principle of change according to which reality operates is a logic embedded in it, the logic in reality; and (2) that what logic really is or should be involves this same real physical-metaphysical but also logical principle. The major components of this logic are:

- A foundation in the physical and metaphysical dualities of nature;

- Its axioms and calculus intended to reflect real change;

- The categorial structure of its related ontology;

- A two-level framework of relational analysis.

Details of the axioms, calculus and semantics of LIR are provided in [8]. Its most important concepts are that (1) every real complex process is accompanied, logically and functionally, by its opposite or contradiction (Principle of Dynamic Opposition; PDO), but only in the sense that when one element is (predominantly) present or actualized, the other is (predominantly) absent or potentialized, alternately and reciprocally, without either ever going to zero; and (2) the emergence of a new entity at a higher level of reality or complexity can take place at the point of maximum interaction between the two.

LIR applies to processes, in a process-ontological view of reality, to trends and tendencies, rather than to 'objects' or the steps in a state-transition picture of change. Processes are described formally as transfinite chains of chains of chains, etc. of alternating actualizations and potentializations of implications, considered with the other logical operators, conjunction and disjunction as real processes themselves. The directions of change are either (1) toward stable macrophysical objects and simple situations, the result of processes of processes, etc. going in the direction of a "non-contradictory" identity or diversity: or (2) toward a state of maximum contradiction (T-state for included third term) from which new entities can emerge. LIR is, therefore, a logic of emergence, a new non-propositional, non-truth-functional logic of change.

Standard logic underlies, rather, the construction of oversimplified models which fail to capture the essential dynamics of biological and cognitive processes, such as reasoning. LIR does not replace classical binary or multi-valued logics but reduces to them for simple systems and situations. In contrast to standard logics, LIR has no difficulty in accepting inconsistency, interpreting it as a natural consequence of the underlying oppositions in physical reality. In summary, LIR is a form of scientific realism in which relations are as fundamental as entities, excluding the abstract entities of classical propositional logic. The terms of those relations, the different degrees of actualization, potentialization and T-state can be regarded as non-standard, non -Kolmogorovian probabilities for which, however, a 
rigorous mathematics awaits development. The most fundamental metalogical as well as metaphysical principle of LIR is that of opposition or antagonism, without which, in this view, nothing could exist.

In LIR, the connectives, that is, what is usually defined as the symbolic logical operators of implication, conjunction and disjunction, all correspond to real operators on real elements in the evolution of real dynamic processes. Accordingly, these operators are, also, subject to being actualized, potentialized or in a T-state. They operate not on theoretical states-of-affairs or propositions, considered as the abstract meaning of statements, but on events, processes and properties, where properties also have the character of processes. The function and process information operators in Burgin's General Theory of Information [3] provide the basis for a more formal characterization of the calculus developed by Lupasco and outlined in [8]. The LIR operators are thus subject to the same logical rules, fundamental postulates and formalisms as other real and hence, natural processes. This answers a potential objection that the operations themselves would imply or lead to rigorous non-contradiction. Real processes are, accordingly, seen as constituted by series of series of series, etc., of alternating actualizations and potentializations. These series are not finite, however. In reality, processes do stop, and they are thus not infinite. This point is significant for the new epistemology to which LIR leads, since it provides the basis for ending infinite regresses in reality. Following Lupasco, we use the term transfinite for these series or chains, which are called ortho- or para-dialectics.

Consequently, the terms of processes are described in LIR as developing into a transfinite series of disjunctions of implications. Every implication is related to a contradictory negative implication in such a way that the actualization of one entails the potentialization of the other and that the non-actualization non-potentialization of the one entails the non-potentialization non-actualization of the other. This leads to a tree-like development of chains of implications, which represent the form of evolution of all complex processes. This development in chains of chains of implications must be finite but unending, that is, transfinite. It is a principle of the Lupasco system that both identity and diversity must coexist, to the extent that they are opposing dynamic aspects of phenomena and consequently subject to its axioms. As we will see, this conception of the evolution of processes corresponds to the description of the underlying microprocesses by Deacon of the incessant flux of micro-contragrade dynamics, without which higher-order orthograde processes and the hierarchy of emergent levels of dynamics would not exist.

\subsubsection{LIR and Other Logics}

It is difficult to compare LIR directly with other logics, including non-classical ones, since the latter deal with the truth or falsity of propositions or their mathematical equivalents, even in their most modern dynamic, modal or doxastic versions. For example, LIR resembles paraconsistent logics (PCL), in which the law of non-contradiction fails. PCL are defined such that contradiction does not entail triviality. According to the LIR axiom of conditional contradiction, however, if A and non-A are present at the same time, it is only in the sense that when $A$ is (predominantly) actual, non- $A$ is (predominantly) potential.

LIR is perhaps closest to quantum logics since its elements are similar to nonstandard probabilities, which do not follow the laws of commutation or distribution. Diederik Aerts' formalism [19] converts quantum mechanics into a system that can be applied to certain macroscopic phenomena, including 
some aspects of the emergence of biological form and human cognition. In the relatively new form of quantum logic that applies here, standard connectives themselves take on new, non-classical meaning, suggesting that, as in LIR, there is a close relationship between logic and physics. Unfortunately, however, Aerts has not attempted to apply his logic to complex processes, such as information.

\subsection{Logics in Incomplete Nature}

Deacon refers to a number of different logics in his presentation. The following is a partial listing:

- logic of emergent dynamics;

- autogenic logic;

- tangled dynamical logic;

- general logic of change;

- theme-and-variation logic;

- teleodynamic logic, e.g., in thought;

- logic of constraint generation, etc.

Using the LIR approach, one can move from these conceptions of logic as a more or less organized body of knowledge or sub-theory to a more formal system of thought. Logic in Reality is formal to the extent that it has axioms, a categorial ontology and something like a semantics that as noted permit stable patterns of inference. In Logic in Reality, to repeat, the inferences are not about the truth and falsity, partial or complete, of statements or sentences, but about the direction of evolution of the components of real processes. With further development, it could offer a methodology that could accurately distinguish correspondence relationships in complex processes and see how they could be interpreted.

For purposes of discussion and debate, I state my position as follows: Logic in Reality "is" the logic of downward causation, the logic of teleological processes, in fact the logic of all emergent dynamics. "Is" here does not imply identity; it rather refers to the two interacting components at the level of both physics and theory that are involved in the process, for example, an overall form and one of the individual sub-processes which will evolve in a "saw-tooth" manner of alternating predominant actuality and potentiality. It provides a logical interpretation of the "entropy ratchet" assumed by Deacon to account for the maintenance of an emergent structure prior to its elimination by diffusion.

As one example, I point to the creation of the document you are reading: the process of its creation has cycled between more or less involvement of the principles of LIR itself, but it has been possible to infer that with the input of the necessary "energy", its potential acceptance could become actual. At this point, its refutation or falsification is potential, but this is a consequence of the application of LIR principles themselves. As I noted in [8], LIR does not contradict or weaken Popper's principle of falsifiability. It complements it by stating that any theory whose argument depends on the absolute independence of the entities or interpretations under discussion may be biased in favor of one other, resulting in errors or omissions. For itself, LIR avoids this trap because it assumes the existence of a counter-theory with which it is necessarily in a dialectical relationship. Reality, for LIR, includes the existence both of LIR and anti-LIR and their conjunction.

I conclude this introduction to LIR by asking the reader, when a reference to it is made in what follows, to try to imagine a "countercurrent" movement of actuality to potentiality, presence to absence 
and vice versa, as describing what is "going on" in the reality of the process. This "logic of energy" accomplishes a number of the tasks set by Deacon as prerequisites for the general application of causal role of absence to the most complex processes available, that is human cognition, behavior, creativity and ethics.

There is thus a broad formal and conceptual parallelism between Deacon's approach and LIR. LIR also sees things in emergent dynamic terms, without the need for any new fundamental law of physics, but the Deacon description is more complete. To repeat, Deacon's innovation is to have emphasized the role of work in the explication of the evolution of complex processes the ability of orthograde and contragrade processes to change the state of things, that is, to do work. The convergence, again, is due to the importance given in both approaches to relations, described more accurately perhaps in LIR as non-separable.

\subsection{Differences between the Deacon and LIR Theories}

There is a broad formal and conceptual parallelism between Deacon's approach and LIR. However, it is important to spell out clearly the differences between them. LIR sees the processes of the world in emergent dynamic terms, without the need for any new fundamental law of physics. It is a philosophical logic (or logical philosophy) and can be and has been criticized as "speculative”. My counter has been that I have, I have tried to apply, as rigorously as possible, the requirements of my own logic, which is grounded in science (physics), and grounds an ontology. However, it is clearly not science and cannot be judged by exactly the same criteria.

On the other hand, the Deacon description is more complete, and is directly grounded in experimental work in neuroscience and evolutionary biology. Deacon's innovation is to emphasize the role of work in the explication of the evolution of complex processes by the ability of orthograde and contragrade processes to change the state of things, that is, to do work. The direct application made by Deacon of the concepts of absence related to entropy (of all kinds) to the formal requirements of a theory of information stand without reference to LIR as such. Nevertheless, the importance given in both approaches to relations, described more accurately perhaps, in LIR as non-separable interactions demonstrates the possibility of a convergence of the perspectives. I hope to show this in the next Sections, where more direct specific comparisons of Deacon's concepts and those of LIR will be made.

\section{The Figure-Background Shift. Form}

In this short Section, I introduce one of Deacon's strongest conceptions in IN namely, that of the inversion of perspective-paying attention to what is not present or predominant in a phenomenon. The term used more frequently by Deacon is that of a figure/background reversal: "A counterintuitive figure/background reversal, focusing on what is absent rather than present, offers a means to repair some of the serious inadequacies in our conceptions of matter, order, life, work, information, representation, and even consciousness and conceptions of value.”

Deacon believes that once this figure/background logic of analysis, this "shift of focus", becomes assimilated into one's thinking about biological, psychological, and semiotics problems, the path toward solutions in each of these domains will become evident. These paths have not been followed 
previously simply because they were not even visible within current paradigms, "in the flat materialistic perspective that has dominated thinking for much of the last few centuries”.

In the alternate paradigm of Logic in Reality two principles support and expand this inversion of perspective, placing it in the context of the well-known concept of Gestalt psychology. The first principle is Gestalt theory is that of an interacting figure, a form or process in a foreground, which stands out against a background or 'ground'. The Gestalt psychologists determined empirically that one never perceives isolated elements that are not somehow combined or associated into perceptions and objects and that, further, any modification of either figure or ground modifies the entire ensemble- $\mathrm{a}$ form.

The figure-ground (background) duality as a structure in reality is easily incorporated into LIR that sees as real all the phenomenological characteristics of structures, in particular, the dynamic phenomenological shifts in perception that take place between figure and ground. Seeing that figures and grounds are related contradictorially, that is, alternately actualizing and potentializing one another, relates them to the processes of which they are the physical and logical consequence. Elements never just "come together" to generate an isolated form. Rather, since every element is itself a form, it is apprehended in the form in which it is included, and every form distinguishes itself as a form, in relation to the form that surrounds it, that is, the ground.

The second principle is that form and structure are not static, inert entities but processes, whether ontological or epistemological. Among the other differences between LIR and Deacon's approach, I note the general absence of process language in the latter. Forms of theories, models or explanations are always undergoing change. Any individual structure or form is totally absolute in any sense, given the nature and logic of energy. It is a dynamic structuring that is always functionally associated with an antagonistic and contradictory potential structuring.

For Lupasco, the energetic dynamisms that constitute all matter and all existence, and the systems they generate, are "pure structural forms, containers of containers, structures of structures, subject to an essential and ineluctable chain of transformation. There is therefore no such thing as a full and static form; devoid of a present, going always from past to future, or even inversely, temporality is immanent to form”. Every form and every structure is thus in LIR terms a real process [20].

The figure/background reversals discussed by Deacon, involving different aspects of the absence-presence duality, are critical to his accounts of organization and, especially, of information. LIR calls attention, in this connection, to the importance of seeing the movement from absence to presence as an equally essential aspect of the dynamics of change.

The figure/ground inversion is one example of the inversions of perspective that are important in other areas of philosophy and science. Logic in Reality explicates the alternating emphasis on one or the other aspect of the phenomenon and the mental change that accompanies it. Examples are the duality between appearance and reality, which is also mentioned by Deacon in connection with the appearance of emergent processes as completely spontaneous, where they are in fact dependent on lower-level processes in a way that LIR describes. 


\section{The Metaphysics of Incomplete Nature}

Let me now restate the assumptions and motivation behind this paper: LIR is a logic, albeit a new kind of logic, that links energy, form and information. Due to its own rigorous ascent from the properties of matter-energy, it provides a way of reconciling the logic of physical science and the logic of living and mental teleology. It offers a new approach to the nature of physical causality and a way to use potentialities to answer the question about getting teleological properties from unambiguously non-teleological starting points.

In this Section, additional key features of Deacon's theory will be presented, with reference to the LIR concepts that support or amend them. The subjects, as will be seen, have the appearance of abstract metaphysical dualities, of interest to philosophers. However, their understanding as science and logic is the objective of this paper, and in both LIR and IN there is a clear convergence of physics and metaphysics. Understanding of the way in which these subjects are treated by Deacon is in my view essential for real progress in the understanding of information, or better information processes, as they operate in the real world.

\subsection{Reciprocity and Complementarity}

Both reciprocity and complementarity have well-defined meanings in mathematics and physics respectively, since Bohr's use of the latter in quantum mechanics for the relation between particle and wave properties of quantum objects. Without going into the historical controversies around these terms, I will use reciprocity to refer to the values of potentialization and actualization of the opposing processes in a phenomenon.

The concept of reciprocity is a key point of intersection of the LIR and Deacon approaches. Reciprocity is the critical complexification mechanism, operating in a vast configurational space of interacting processes, that insures the emergence of higher levels of ortho- and contragrade processes. In Deacon's system, synergistic reciprocal interaction is essential for the emergence of the next higher level of complexity. But such reciprocity can only exist if the system has the capacity or potential, full or residual, for it, as yet "unsaturated”, to use Frege's term. Reciprocity therefore implies potentiality as well as actuality. Logic in Reality establishes the existence of this duality as a principle at the lowest ontological level, thus grounding reciprocity between more complex process entities. In this interpretation, dualism or duality is not a block to emergence; it is its sine qua non.

Reciprocity refers in Deacon's system to a specific form of mutual constraint generation, the special systematic form of limitation that enables the material and energetic threads of the physical universe to become entangled with one another in just the right way so as to produce the additional higher dimension of causal processes that is the fabric of life and mind. The critical molecules constituting an organism are enmeshed in a process of reciprocal synthesis, in which each is the product of the interactions among many others in the system. They exist and their specific properties are created by one another as a result of this higher-order systemic synergy.

LIR agrees with this conception of reciprocity, but it requires an answer to the further question of the properties of the smallest molecules of which the larger ones are composed. The other point is that chemical reactions involve to an overwhelming degree only two molecules at a time, despite their 
location in vast networks, including catalysis by active sites of both complex super-molecules or surfaces. For such reactions, one must have available the necessary potentialities, capacities to enter into the reaction in the first place. It is with regard to these potentialities and their synchronic and diachronic involvement in change that LIR is able to provide a logical description.

Deacon refers to potential in pointing out that the complementarity and reciprocity of morphodynamic processes produces a special kind of emergent stability, unavailable to either process in isolation. Indeed, for all real complex biological processes, the entity in question and the environment are reciprocally defined and determined with respect to each other, because the same molecular configuration in a non-supportive environment lacks any of the defining properties of autogenesis.

The concept of reciprocity also enters into the linkage between forms of work and constraint in the interpretive process that is involved in information (see Section 7). Unlike a thermostat, where the locus of interpretive activity is extrinsic to the cycle of physical interactions, an interpretive process is characterized by an entanglement between the dynamics of its responsiveness to an extrinsic constraint and the dynamics that maintains the intrinsic constraints that enable this responsiveness. Information is in this way indirectly about the conditions of its own interpretation, as well as about something else relevant to these conditions.

Logic in Reality, again, is about the physical dynamic structure of an entanglement between the components of processes of this kind. Even in a model autogenic system, each part (itself a morphodynamic product) is involved in an ultimately closed set of reciprocal interaction relationships with each other to create the whole, but it takes the whole reciprocally synergistic complex to generate each part. In standard logic, this would amount to a logical-type violation (in which a class can also be a member of itself). In Logic in Reality, however, the ontological feature of non-separability provides the conceptual framework for the relationships. By including the capacity to model itself in relation to extrinsic features of the world, a neurally generated teleodynamic system similarly introduces a higher-order tangle to this dynamical hierarchy.

Logic in Reality establishes an ontology of complementarity based on the Principle of Dynamic Opposition as a "meaning principle". The necessary concepts are the non-separability of individuality and non-individuality; part and whole; subjectivity and objectivity in relation to the experiment-experimenter pair; and of object and meta-levels of theories. The difference, which is not absolute between reciprocity and complementarity is only that the former is more quantitative and the latter more qualitative. Deacon discusses complementarities of symmetry and shape as the source of self-amplifying non-linear processes and also refers to the "reciprocal complementarity of these self-organizing processes.”

In evolution, organisms embody vast webs of constraints in their internal dynamics, and specifically with ever more diverse means of interacting with their surroundings, they have increased the ways in which they are able to impose these or complementary constraints on the dynamics of external events and relationships. So the functional properties of life only emerge when homeodynamic and morphodynamic processes are organized in precisely complementary and completely reflexive ways with respect to one another. This use of the principle of complementarity by Deacon is justified by the LIR interpretation. 


\subsection{Circularity or Spirality?}

Since Varela wrote his famous article on autopoesis [21], illustrated with Escher's cartoon of two hands drawing each other, it has been customary to associate autopoesis with circularity. At the same time, as we all know, no real process, as expressed in the 1st Axiom of Non-Identity of LIR, returns to the same place, although many simple ones do to all intents and purposes. For complex animate systems, however, the concept is clearly inaccurate, and one can wonder why the more correct idea of a spiral evolution is not used. I believe this is another expression of the resistance of human beings to accept absence, here equated with non-identity, as ontologically valid.

I have accordingly rephrased here the statement in $\mathbf{I N}$ about the individuality of organisms: it is the spirality of the consequential architecture-teleodynamics - that both delineates and creates the individuality that is the organism itself. This spirality is found in the simple changes of the inorganic world, but they are not self-reflexive. Having a brain also entails the generation of a form of teleodynamic relationship that is partially organized with respect to itself as environment. However, this higher-order form of teleodynamic causality, which creates an entirely novel emergent realm of self-dynamics, is not, does not need to be and should not be described as circular. The concept of spirality is equally if not more explanatory as to why being an organism with a complex brain inevitably involves a doubly reflexive, dyadic organization rather than simple bi-univocal relations.

As Deacon points out, further, the distinctive higher-order form of teleodynamics that emerges from a teleodynamic process must include itself as a component, but that the very locus of teleodynamic closure becomes virtual does not require circularity. It is a tangle in the dynamical hierarchy that is not superficially analogous "to the part/whole tangle that defines teleodynamics more generally", but strictly analogous. In complex systems, each part (itself a morphodynamic product) is involved in an ultimately closed set of reciprocal interaction relationships with the others to create the whole, but it takes the whole reciprocally synergistic complex to generate each part. Deacon correctly states that in (standard) logic, this would amount to a logical-type violation (in which a class can also be a member of itself), Logic in Reality avoids the aporia because "becoming virtual (or potential)" does not require total virtuality, and in the mereology of LIR, parts and wholes also share, contradictorially, some of one another's properties.

There is an additional property of spirality that brings us immediately closer to the real world-its handedness. While it is not a direct property of energy as is the case with the up and down "spin" of electrons, spirals or helices must be left-handed or right-handed, mirror images that can nevertheless be distinguished by the way in which they polarize light. They share this property with the basic minimally asymmetric simple molecules, sugars and amino acids that compose the more complex macromolecular structures of life.

\subsection{Absence and Quantum Logic}

Deacon is clearly aware that a new logic of quantum-level and relativistic processes is needed to explain some of the paradoxical aspects of those processes that currently available quantum logics do not [20]. But for the purpose of making sense of the teleological features of life and mind, he states that the physics itself of extremes of scale in space, time and energy are not relevant as such. Deacon's 
contribution has been to show that one need go no further than classical statistical mechanics to make sense of these phenomena.

Although Logic in Reality is a more modern scientific paradigm than the pre-relativistic and pre-quantum framework in which Deacon works, pointing to the Principle of Dynamic Opposition that has been ignored in physics, it does not undermine his conclusions. To the extent that quantum logic is relational, as in Relational Quantum Mechanics, the effective potentialization of the entities between which the relations obtain is comparable to "absence".

\subsection{Emergence}

I have referred to emergence and emergent processes on a number of occasions, without detail, but this subject is in fact one to which Logic in Reality brings a number of clarifications. Both Incomplete Nature and Logic in Reality devote substantial sections to the history of emergence which will not be reproduced here, but LIR offers specific answers to the problems remaining with the best current pictures of emergence.

Rather than attempt to argue my position in detail, I will simply state some of my conclusions following from the application of the LIR principles.

(i) LIR deconstructs Kim's argument against emergence by eliminating the absolute separation between levels that it requires. The paradoxical nature of synchronic reflexive downward causation is eliminated by new concepts of related synchrony and diachrony: downward causation from the emergent level to the parts level is extended in sequential time and is a movement through phase space.

(ii) LIR avoids Humphrey's use, referred to by Deacon, of the poorly defined concept of "fusion". In simple physical fusion, processes as well as materials lose their identity, which is not the case at the higher levels of interest.

(iii) LIR improves upon Bickhard’s “interactivism” by replacing his propositional, truth-functional requirements for demonstration of its correct application. In contrast to his statement, "particles participate in organization, but do not themselves have organization”, I point simply to the causally effective potential in the structure of atoms and the molecules that they constitute as necessary and sufficient organization.

(iv) Rather than static notions of part and whole, LIR sees parts and wholes also as dynamic processes in an interactive relation, sharing part of one another's properties.

The LIR theory of emergent processes thus provides alternatives to classical views of part-whole organization and supervenience, not by eliminating these principles but seeing that they must instantiate the ontological feature of non-separability. The relevant contradictorial overlaps that are allowed by the basic principle of dynamic opposition are not only between part and whole, but also between upper- and lower-level properties. In LIR, organization or structure is, also, not a comparative assessment, but a process that is causally efficient in its own right.

In the emergent classes of phenomena, life and human cognition, it is always the residual potentialities as well as actualities at the lower level that provide the causal information necessary for emergence of new structures at the next higher level. The organizations that characterize these 
ententional phenomena do not display "simple" compositionality or mereology, the limitations of which are clear. But processes do have other processes as their parts, when the processes are understood as composed of dynamic sub-processes incorporating different degrees of actuality and potentiality, identity and diversity. As I showed in [8], LIR provides a conception of the space-time associated with the evolution of complex processes that is both synchronic and diachronic, which is necessary for a concept of emergence that can apply to them.

Logic in Reality, then, starting with its physically grounded 3rd Axiom of the Included Middle, is in fact a Logic of Emergence, the emergent transitions of complex dynamic processes and processes of processes that constitute life, etc. LIR is, to repeat, a theory of real processes in which Bickhard's statement that "mental states are processes like flame states" is obvious. It is described in the LIR epistemology as grounded in the principle of dynamic opposition at the level of the logically contradictorial dynamics of nerve cell activation and passivation.

I will now apply all the concepts developed in the above Sections to the understanding of the concept of information which, in view of its manifold aspects and complexity Hofkirchner [22] has called a "super-concept", an emergent phenomenon par excellence.

\section{Information}

Recent work on the theory and philosophy of information has begun to refer to its qualitative, meaningful and value-laden properties in addition to its quantitative measures, derived from communication theory. A unified theory of information is now more possible, and several approaches to one have been proposed [22], many emphasizing an ethical role for information in the emerging "information society" [23]. I have summarized the state of the art from the LIR perspective [24], emphasizing the role in opening up the definition of information to include contradictory and inconsistent aspects in a picture of information-as-process. The concepts of information as a dynamic process "in its own right" and information as a phenomenon inhering in the structure of processes cannot be totally separated. But the problem with these approaches, including LIR, is that they do not give an adequate explanation of the emergence of higher level informational processes in relation to the underlying dynamic processes upon which they supervene.

Deacon's application of his concept of the dynamic role of absence to information constitutes a major step forward in this area. A key paper [5] that appeared prior to IN, entitled "What is missing from theories of information" is summarized here as an introduction to its more extensive discussion in IN.

\subsection{What is Missing from Theories of Information?}

A complete account of the nature of information that is adequate to distinguish it from more simple material or energetic relationships requires a figure/background shift that is even more fundamental and more counterintuitive than that for energy (cf. Section 5.) This is because what matters is not an account of only its physical properties, or even its formal properties. What matters in the case of information, and produces its distinctive physical consequences, is a relationship to something not there. Information is the archetypical absential concept.

Deacon's key concept is that information is a relational property that emerges from nested layers of constraint: constraints of signal probability (Shannon), constraints of the dynamics of signal generation 
(Boltzmann), and the constraints required for self-maintaining, far-from-equilibrium, end-directed dynamics (Darwin). Because information is a relationship among levels of constraint generated by intrinsically unstable physical processes, it is also normative with respect to those processes. As above, constraint is a partly negative as well as a positive property or entity, and thus only partly something intrinsic or determinate; it is intrinsically incomplete and fallible, or in LIR terms characterized by its potentialities. The nested dependencies of the three levels of entropy reduction-characterized by Shannon's, Boltzmann's, and Darwin's variations on this theme of entropy reduction-define a recursive architecture that demonstrates three hierarchically nested notions of information. These three very roughly parallel the classic hierarchic distinctions between syntax (Shannon), semantics (add Boltzmann), and pragmatics (add Darwin). They also roughly parallel the relationship between data, content, and significance. LIR suggests a way of understanding how these semiotic levels are interrelated.

Deacon brings out the differential impact of both Shannon and Boltzmann entropy [25] on the structure of information and information transfer. He identifies two contributors to the entropy of a signal — one associated with the probability of a given signal being sent and the other associated with a given signal being corrupted. This complementary relationship is an indication hint that the physical and informational uses of the concept of entropy are more than merely analogous. By exploring the relationship between Shannon entropy and Boltzmann entropy, one can shed light on the reason why change in Shannon entropy is critical to information. But the connection is subtle, and its relationship to the way that a signal conveys its information "content" is even subtler.

I view Deacon's approach as supporting and extending the insights of Robert Logan and his colleagues expressed in their views on the nature of information in biological systems. Logan begins [26] by recalling Mackay's idea of structural information. Mackay's move was to rescue information that affected the receiver's mindset from a pejorative label as "subjective". He proposed that both Shannon and his followers were concerned with what he called "selective information", that is information calculated by considering the selection of message elements from a set. But selective information alone is not enough; also required is another kind of information that he called "structural". Structural information indicates how selective information is to be understood; it is a message about how to interpret a message - that is, it is a meta-communication.

Structural information (Logan continues) must involve semantics and meaning if it is to succeed in its role of interpreting selective or Shannon information. Structural information is concerned with the effect and impact of the information on the mind of the receiver and hence is reflexive. Structural information has a relationship to pragmatics as well as semantics where pragmatics tries to bridge the explanatory gap between the literal meaning of a sentence and the meaning that the speaker or writer intended.

In their key document [11], Kaufmann, Logan and their colleagues defined biotic or instructional information, defined as the constraints that allow an autonomous agent, i.e., a living organism, to convert free energy into work so that the living organism is able to propagate its organization through growth and replication, is intimately connected with meaning. "For Shannon the semantics or meaning of the message does not matter, whereas in biology the opposite is true. Biotic agents have purpose and hence meaning. One can therefore argue that since the meaning of instructional information is propagating organization that we finally understand the meaning of life: the "meaning of life" is propagating organization. This remark is not meant to trivialize the great philosophical quest for the 
meaning of life from a human perspective but there is a sense in which the meaning of life including human life is indeed the propagation of organization. The purpose of life is the creation or propagation of more life. In addition, Shannon information does not necessarily entail meaning whereas biotic or instructional information always entails meaning. The other essential difference is that Shannon information is defined independently of the medium of its instantiation whereas biotic information is very much tied to its material instantiation in the nucleic acids and proteins of which it is composed."

I will discuss this approach later in the Section on meaning. I add here only that the original concept of structural information can be better stated today in the processual terms of LIR. Structures themselves should not be considered as static entities but as processes themselves, as originally pointed out by Lupasco [18], and information inheres in the structural relationships of the processes as a network of vectors.

Ulanowicz [27] has criticized Deacon's approach by suggesting that its grounding in the 2nd Law of Thermodynamics fails to capture the quantitative dynamic relation between two critical features of a system, its constraint A and what is absent $\Phi$. Ulanowicz designates the former as the apodictic, roughly information, and the latter as the apophatic, which measures the flexibility of a system. Following the 3rd Law of Thermodynamics, like entropy itself, information is always relative or relational. Unless the apophatic aspect and the dialectic between the two components are taken into account, other characterizations of information fail. This development therefore goes beyond Deacon's statement that constraints are not intrinsic properties but relational ones, even if just (sic) in relation to what is possible. Such relations are, exactly, what LIR addresses.

LIR supports the dialectical approach of Ulanowicz to improving the Shannon/Boltzmann mathematics to characterizing and measuring information, eventually to gain deeper insight into the dynamics of nature. However, I have questioned Ulanowicz' use of two linguistic terms in his dialectic, the first (apodictic) generally referring to demonstrable propositions and the second (apophatic) not to that which is not but that which is not or cannot be said. However, by assigning a property of flexibility to $\Phi$, Ulanowicz leaves the door open to a dynamic interpretation, possible with LIR. For example, flexibility, as a property of real materials (and human beings) can be seen as a dialectical conjunction of elasticity and plasticity. The LIR + Deacon conception of the dynamics of absence can be usefully supplemented by that of Ulanowicz, which provides a necessary quantitative component in the search for an "encompassing perception of reality".

The key point is that it is the relation, which follows in Ulanowicz' concept of the 3rd Law, that delivers meaning. As he writes, one of the foremost criticisms of the Shannon approach as generally understood is that it cannot address "meaning" in any realistic way, as the original Shannon "entropy" is a conflation of both constraint and flexibility. Ulanowicz provides a numerical example in which meaning appears as a "pale shadow" of reality, a "wanly quantitative model”. We agree, therefore, that the meaning of real phenomena and their contradictorial properties is primitive. Reflections in mathematical models, as in the Gödel theorems, will be eliminative, unless it is referred to the underlying dynamics (Principle of Dynamic Opposition). Using LIR would permit, therefore such correspondence in a relational context that obviates any absolute disjunctive interpretation by allowing its decomposition into two agonistic (complementary) terms that quantify the degree of system constraint and its residual freedom, respectively. The implication of this decomposition is that the 3rd Law applies not only to the concept of entropy, but to the conjugate constraint as well. That is, 
constraint (and its degenerate subclass, information) has no meaning in abstraction from the 3rd Law. Like entropy, information is always relative. That is, what is measured as constraint or information of $a$ in the context of $b$ will generally be different from that between $a$ and $c$. In other words, Ulanowicz' terms, like the elements of LIR, do not commute. His reinterpretation of Shannon-Boltzmann theory is in terms of a complementarity which LIR simply refines and, literally and figuratively, makes more logical.

\subsection{Referential- and Inferential-Information}

For Deacon, referential information is information that any sign or signal, or, more broadly, any real process provides is not an intrinsic feature, but a function of extrinsically imposed constraints. The referential information is in this sense inferred from a formal characteristic embodied in the relationship between those signal features that are present and those that are absent. This is exactly the LIR picture of the relationship expressed by the Principle of Dynamic Opposition, in which inferences are made from the relationship between features that are predominantly present (actual) and absent (potential), except that no feature is $100 \%$ one or the other. Further, LIR always includes reference to the movement of a process from presence to absence. The statement that the formal characteristics are expressed in the signal in terms of what is not included but could have been, can be generalized to what is not included but still may be (in the future).

Referential information is second-order information, or information about information. It is in a sense inferred from the formal characteristics embodied in the relationship between unconstrained possibility and realized constraint. In this way, Shannon information, which is assessed in terms of this constraint, embodies a trace of the work that produced it. Self-rectifying constraint preservation is the defining criterion of referential information. This property is the foundation for all higher-order intentional processes.

Even the absence of any change of signal entropy can provide referential information. No change in entropy is one possible state. This means that even unconstrained fluctuations may still be able to convey referential information, information about the fact that of the possible influences on signal constraint, none were present. The informative power of absence is one of the clearest indications that Shannon information and referential information are not equivalent.

I further note, applying the principles of LIR, that referential information is also inferential information. If the dynamics of information involve the movement from presence to absence, inferences can be made about that movement. As a further advantage of LIR, it is not necessary, and this is one of the original insights of Lupasco, to assume yet another level of information about information about information, with the specter of an infinite regress. As he showed, the PDO that insures the interaction between two levels exhausts the available information, and none is added by a further iteration. As one observes, potential infinite regresses of epistemological processes, e.g., information or sentience, stop in reality. I conclude that referential or second-order information is a useful approach to defining the characteristics of what I call qualitative information involving human values. I consider this a vital step in trying to reintegrate issues of subjective value into the natural sciences. 


\subsection{Representation in Information}

Among the points for further work indicated in my article on this subject [28], I asked whether the concept of potentiality in LIR, which implies partial absence, can be related to Deacon's view of information and representation as dependent on specific absences in their dynamics. I now believe that Deacon and I come to similar conclusions regarding the concept of representation in information. Deacon for example states that a representational relationship cannot be vested in any object or structure or sign vehicle. It is not reducible to any specific physical distinction, nor is it fully constituted by a correspondence relationship. But neither is it a primitive unanalyzable property of minds. Instead, even simple functional and representational relationships emerge from a nested interdependence of generative processes that are distinctive only insofar as they embody specific absences in their dynamics and their relationships to one another. These absences embody, in the negative the constraints imposed on the physical substrates of signals, thoughts, and communications that can be transferred from one substrate to another, and which thereby play efficacious roles in the world as inherited constraints on what tends to occur, rather than acting as pushes or pulls forcing events in one direction or another. As noted previously, constraints do not do work, but they are the scaffolding upon which the capacity to do work depends.

In a Section in $\mathbf{I N}$ entitled the Thermodynamics of Thought, Deacon asks: "So what would it mean to understand mental representations in terms of global homeodynamic and morphodynamic attractors exhibited by vast ensembles of signals circulating within vast neuronal networks? Like their non-biological counterparts, these higher-order statistical dynamical effects should have very distinctive properties and requirements. But now consider what it would mean for a teleodynamic process to include within itself a representation of its own dynamical final causal tendencies. The component dynamics of a teleodynamic process have ententional properties precisely because they are critical to the creation of the whole dynamic, which in turn is critical to the continued creation of these component dynamics. Were the reciprocal synergy of the whole dynamic to break down, these component dynamics would also eventually disappear. The whole produces the parts and the parts produce the whole. But then a teleogenic process in which one critical dynamical component is a representational process that interprets its own teleodynamic tendency extends this convoluted causal circularity one level further."

Simply asserting the existence of a mapping between properties in the world and properties of a mechanistic process presumed to take place in the brain implicitly frames the representational relationship in classically dualistic terms. Everything depends on the nature of the correspondence, and specifically, what makes it different from either an isomorphic or an arbitrary one-to-one mapping between components or phases of two distinct processes. Logic in Reality provides an alternative to such a simplistic mapping by emphasizing the physicality of the relation.

Deacon concludes that in the exploration of the relationship between information theory, thermodynamics, and natural selection, some of the unrecognized complexity hidden within the concept of information can be seen. By generalizing the insight captured by Shannon's equation of information with entropy reduction and constraint propagation, and tracing its linkage to analogues in thermodynamic and evolutionary domains, Deacon has addressed some of the most vexing issues of representation, reference, and normativity. "By removing these inadequacies in current definitions of 
information, we may at last overcome the seemingly insurmountable obstacles to formulating a theory of representation that is sufficiently rich to serve as the basis for biology and the cognitive neurosciences, and sufficiently grounded in physics to explain representational fallibility, error checking, information creation, and the relationship between informational and energetic processes."

In my view, Deacon's conclusions are justified, subject only to the need for the inclusion of the necessary physical PDO to ground thermodynamics itself and hence all phenomena at higher levels of reality. These are typified by human cognitive processes as will be discussed in the next and final Section.

\section{Intentionality, Consciousness and Meaning}

\subsection{The Calculus of Intentionality}

In $I N$, Deacon summarizes his view of the functional role of teleodynamic organization as the most primitive physical system "capable of influencing other physical systems via something that is merely virtual - that which is specifically absent, missing displaced, potential or merely abstract.” In the thought experiment adduced in support of this concept, he shows that its emergence is enabled by the premature halting of the component morphodynamic processes, "a tendency that is spontaneous and would otherwise run to self-extinction, that makes self, information and life (N.B.: that is, the loci of intentionality) possible." Deacon invokes a mechanism of an "entropy ratchet” that permits the brief buildup of constraints by morphodynamic processes and the halting of the process before there is any dissipation of those constraints. "The possibility of this occurring is the secret of this distinctive form of causality. In principle, it allows the generation of constraints to continually reconstitute this intrinsic potential endlessly. It also provides the capacity to remember and reproduce information, because self-rectifying constraint preservation is the defining criterion of referential information. This property is the foundation for all higher-order intentional processes.”

In the light of the principles of Logic in Reality, outlined in this paper, I believe that several amendments should be made to the above paragraph, as follows:

(i) The characterization of "virtual" should be modified to what is predominantly absent, displaced or potential. Abstract systems do not interact physically with other physical systems.

(ii) The premature halting that is displayed by morphodynamic processes cannot be in and of itself spontaneous, but requires (1) the existence of some opposing process and (2) the potential or capacity of the first process to interact.

(iii) The entropy ratchet described, without dynamics of its own is a perpetual motion machine; "continually" and "endlessly" are terms that cannot be ascribed to real processes. The calculus of LIR describes the evolution of real processes in terms of a "saw-tooth" movement between actuality and potentiality is not idealized, but permits a largely if not completely perfect reconstitution of constraints. The capacity for self-rectification requires, but can be traced back to, residual unsaturated potential of the basic physical components of the entities involved.

Logic in Reality supplements Deacon's picture since it is not the scalar features of energy (extension, conservation) that are involved in dynamic opposition at the macroscopic level of life and 
mind, but something like the intensive self-duality of quantum entities which become the familiar physical dualities in the microscopic thermodynamic domain on which the macroscopic supervenes.

Distributed, non-localized potentialities as well as actualities are the carriers of information necessary for the emergence of entities at higher levels of reality or complexity. The residual, unsaturated charge on an amino acid has its counterparts at higher levels (no homunculus necessary). At the current state of knowledge about deep quantum levels, all the correlations and constraints provided for in both Deacon and LIR fail. They are valid only for the thermodynamic domain, not the "timeless" one of the quantum vacuum, studied by the biologist Michael Conrad. In the absence of a demonstration of direct coupling between the sub-quantum levels and biology, which neither Conrad nor his successors have proposed successfully, Deacon's position holds.

The purpose of Deacon's Incomplete Nature is not to ground the attributes of living systems in contemporary physics and chemistry, but to focus attention on a more general problem of accounting for what amounts to a phase change in dynamical organization that necessarily occurred with the origins of life, based on its essential absent features, irrespective of any specific molecular details. Deacon states, however, that the chemistry of living systems involves a contragrade chemistry, and as indicated above, contragrade processes are contradictorial in the LIR sense, involving movement reciprocal movements from actuality to potentiality of their components. Thus what LIR brings to this picture is to define the relevance of the known absential organization in atoms and molecules, their "potential", to these dynamic changes and which the dynamic changes also instantiate. LIR is, thus, a logical bridge that eliminates a conceptual discontinuity that Deacon has accepted, albeit provisionally. The reason that chemistry can have this absential property is indicated above. Chemistry by itself does not only naturally follow the 2nd Law of Thermodynamics to the letter, but depends on the constantly present operation of the Pauli Exclusion Principle in a sense contrary to that of the 2nd Law.

The relationships between neurally embodied forms of homeo-, morpho-, and teleodynamic processes and various aspects of mental experience are discussed in detail in $\mathbf{I N}$, and I will not go into them further here. Deacon's reference to traces of the structure of experience establishes a relation to the logical view of experience in LIR. This is the sense in which Lupasco's dictum "Logic is experience; experience is logic” is to be understood.

\subsection{Sentience and Consciousness}

The core hypothesis of $\boldsymbol{I N}$ is that all teleodynamic phenomena necessarily depend upon, and emerge from, simpler morphodynamic and homeodynamic processes. This implies that the complex intentional features that characterize our thoughts and subjective experiences must likewise emerge from a background of neurological morphodynamic and homeodynamic processes. Moreover, these lower-order subvenient dynamical features must also inevitably constitute significant aspects of our mental lives. It is impossible to even approach issues of sentience without taking the necessary contributions of homeodynamic and morphodynamic aspects of mental experience and brain function into account. Deacon has thus reframed the concept of sentience in emergent dynamical terms which allows the addressing of questions that are not often considered to be subject to empirical neuroscientific analysis. He believes that these phenomena are entirely available to scientific 
investigation once we discover how they emerge from lower-level teleodynamic, morphodynamic, and thermodynamic processes.

The so-called hard problem of consciousness was reconceptualized in other dynamic terms by Lupasco which nevertheless allowed him to differentiate between different kinds of consciousnesses and unconsciousnesses as functional processes in a more rigorous manner than had been possible for a Freud or Jung [29]. As Deacon says, what appears to make the hard problem hard is our predisposition to frame the problem of consciousness it in mechanistic and computational terms, presuming that its intentional content must be embodied in some material or energetic substrate. This amounts to a reification of consciousness, claiming that it is something different and separate from the energetic relationships in which it is instantiated.

LIR thus supports the Deacon program. The "how" of the emergence is certainly not completely in hand, but LIR provides an additional critical property of the form of the interactions involved, including the emergence of new ideas through the opposition of conflicting drives in an individual's mentality. It may be objected to the above theory that nowhere does it provide an explication of individual awareness. This is correct, but such an explication is not to be found in any other current theories of first-person approaches to the study of consciousness, for example, that of Varela and Shear in their review volume [30]. In their terms:

"The received distinction between objective and subjective as an absolute demarcation between inside and outside needs to be closely scrutinized. Mutatis mutandis, dealing with subjective phenomena is not the same as dealing with purely private experiences, as is often assumed. The subjective is intrinsically open to inter-subjective validation, if only we avail ourselves of a method and procedure for doing so (emphasis ours)".

The LIR ontology provides a realistic basis for an interaction between subject and object, eliminating the absolute characteristics of both. The LIR position is that the concept of the isolated individual is a convenient fiction, an appearance resulting from the high degree of survival value it confers. LIR, however, suggests that it is scientifically justified to include first-person, subjective experience as an explicit and active component of a science of consciousness.

I am aware of one weakness in this argument: as all will admit, the emergence of individual human consciousness can be described in terms, for example, of increasing levels of complexity of brain function providing selective advantage, but this is not an explanation. One can also say, in terms closer to Logic in Reality, that because consciousness exists, there was the potential for it at all times since the origin of the universe. The reason that this statement escapes being a meaningless self-evident tautology is that it points to the patterns of evolution from potentialities that are easier to see as real at lower physical, chemical and biological levels of reality. Its statement in LIR terms is consistent with the concept of hylozoism (matter as somehow animate even if not conscious) discussed by Stanley Salthe and others but more grounded in physics, in terms of potential energies.

This paper is a first attempt to support the basic formulation of the application of absentialism by Deacon to cognitive issues on a logical basis. One can, I hope, better discuss reality when one can assign appropriate features to presence and absence, existence and non-existence. One can also make a start on discussion of the even "harder" problem of the specificity of individual consciousness, why I am this illusion (for people who think in terms of illusion), rather than another. At the present time, this problem will have to be relegated to the less accessible areas of metaphysics. 
The discussion of the finality of sentience, for example, needs obviously to be taken beyond the "negative" good of the avoidance of individual or group suffering. As Luhn has pointed out, without making any transcendental judgments, it is obvious that people seek knowledge and understanding, not for the sake of immediate gain or satisfaction of basic needs but for the common good. However, achieving real understanding via the "golem" of the Internet, in what Nicolescu has called "Cyber-Space-Time”, is by no means obvious. The contradictorial principles of Logic in Reality would suggest that the current, again, idealization of the value of a globalized post-industrial information society, with its center of gravity in the North and West should and will be followed by a return to a more balanced sharing of global resources.

A third finality of sentience is the achievement of increased knowledge as a religious imperative, for the sake of a deity. I will not discuss this question here except to remark that it can be seen as another example of the figure/ground problematic discussed above. Of the everyday world and the "other" world, which is the reality, and which the appearance, a "creator" or the "created"?

On the other hand, without going outside the domain of science, I can point to the existence of human creativity and its products - art-as evidence of a form of purpose that is self-rewarding as well as justified in terms of a common good. Art is thus another emergent phenomenon, for which the underlying dynamics are the conflicts and drives in the mind of the artist. As indicated above for information, it is not necessary to worry about the possibility of yet another level of sentience of sentience of sentience, with the specter of an infinite regress. The principle of dynamic opposition that insures the interaction between two levels exhausts the available information, and none is added by a further iteration.

\subsection{Cybersemiotics and Meaning}

In his book [13] and one of several key papers [31], Sören Brier proposes a cybersemiotics, the inadequacies of all standard attempts to arrive from a physico-chemical scientific paradigm, an informational-computational materialism, at the phenomena of first person subjective consciousness and meaning. His approach shows the necessity of uniting approaches from physics, biology, phenomenological awareness and intentionality and socio-linguistics in a transdisciplinary theory of information. For Brier, standard scientific concepts of meaning cannot explain meaning from a bottom-up approach. "The meaning of information is not information and information of meaning is not meaning, when we only use the term information in its physicalist sense."

Brier's conception of information and meaning is, very much in the spirit of Logic in Reality, to see them as non-separable, so that qualitative properties emerge from something, a relation fundamentally present $a b$ origine and not from a simple causal hierarchy. In LIR, of course, this something is no more mysterious than the residual potentialities of the system.

I am thus in accord with Brier's critique of various very well-known attempts to develop the bases of meaning in information including systems theory, the autopoesis of Maturana and Varela, and the sociology of Luhmann. I disagree only with the strategy adopted by Brier and also to some extent by Deacon of basing biosemiotics and cybersemiotics on the transdisciplinary (his designation) semiotic philosophy of Peirce. I have outlined my position in [28]; basically it states that the interpolation of a category of signs as entities between phenomena and their interpretation by human beings is valid as a 
method of classification but does not reflect the ontological dynamics of those phenomena. Thus, I have not chosen to develop a notion of meaning (or information) from standard semiotics since I feel that they fail for two fundamental, related reasons: (1) they do not have, and have no basis for having, a way of distinguishing between signs that are inert symbols or symbolic operators; and (2) they provide no basis for signs corresponding to absent or potential aspects of phenomena. Both failures can be traced back to the original limitations consequent on Peirce's avoidance of opposition in his fundamental metaphysics and his insistence on "blind" chance and absolute continuity as fundamental properties of the functioning of the universe.

The application of the principles of Logic in Reality, then, is a non-reductionist methodology for enabling physics to be "more fundamental" than the other sciences, while allowing evolutionary experiential and intersubjective processes be the ground reality of human existence in the sense of Heidegger [32].

\subsection{Meaning as Process. Significant Information}

Deacon does not, in $\mathbf{I N}$, explain in detail what he means by meaning, but he does make numerous references to it as a phenomenon of the same kind as consciousness, function, purpose and value. These teleodynamic ententional phenomena are defined by their incompleteness or absential character, by what is not "there in any typical sense of being materially or energetically embodied, and yet may still be causally relevant". "In each of these cases, there is something present that marks this curious intrinsic relation to something absent." If one parses these statements, one must say that in the case of meaning, there is something present (which is not meaning itself) that marks the relation to something absent (which is not itself meaning). This something is, of course, the result of the operation of a real, physical constraint, and meaning is to be understood as existing as a consequence of this complex of relationships.

In my opinion, Deacon has made life (sic) difficult for himself by excluding (or bracketing) the materiality of meaning as a real process, that is, one which is "energetically embodied" in the language of LIR, and whose dynamics follow the schemes that Deacon establishes for other emergent phenomena. One may then look at meaning, like information as constraints and constraints as meaning, emerging in the process of the active interpretation of those constraints by human agents.

Meaning can be related to our overall theories by defining it as significant information. For Deacon, significant information is what emerges at the third level of complexity at which "Darwinian" forms of interaction occur, and such information has meaning for the organism both in the sense of usefulness for survival and the accomplishment of higher level cognitive functions. In other words, evolution has "provided" sufficient complexity for complex feedback loops such that meaning becomes equivalent to information relative to survival.

A full discussion of meaning requires an epistemology, an understanding of the relation of the thought processes leading to meaning and a dynamic picture of how we know. In both the Deacon and LIR approaches, meaning is an emergent phenomenon, of which I attempted a picture in terms of levels [12]. In the epistemology of LIR, the principle of dynamic opposition, applied at all levels to the various entities considered as processes defines a hierarchy in terms of complexity of interaction, both internally and externally. 
As noted, Stuart Kauffman, Robert K. Logan and their colleagues also proposed a new reading of information that unites matter, energy and information [11] that is consistent with this picture. They show that neither the Shannon definition of information as a scalar quantity of bits, devoid of meaning, nor Kolmogorovian information which refers to standard probability distributions of non-interactive systems is applicable in biology. Information should be designated as 'instructional' or 'biotic' in the sense that it carries meaning and consists of constraints or their physical equivalents-boundary conditions that are the causes of events. Most importantly, the coming into existence of the constraint is itself part of the propagating organization of the entity. "Constraints are information and information is constraints.” This recursive aspect is characteristic of non-Markov chains, the non-Kolmogorovian probability behavior of two mutually dependent entities, better processes, to which LIR applies.

Accordingly, one can write:

Information (quantitative; Shannon/Weaver) $\rightarrow$ Meaning $1 \rightarrow$ Knowledge (qualitative; Knowing: Kauffman/Logan Intensional or "Biotic” Information) $\rightarrow$ Meaning $2 \rightarrow$ Knowledge of Knowledge (or Knowing) $\rightarrow$ Meaning 3 .

Meaning 1 is inherent in information as a constraint/potentiality at non-cognitive levels of reality. Leydesdorff calls this a "first codification". Knowledge enables codification of the Meaning 1 of information, and this knowledge can be further codified.

At the cognitive level, information (and its meaning) is organized into knowledge and acquires Meaning 2 that subsumes normative and other values. LIR applies to both Meanings 1 and 2, since the origin of the former is also in basic physics. Knowledge is however more than just a classification; it is also a process of "knowing”, not reified. Meaning 2 will be present not only with knowledge but also within the process of knowing (human and animal). At the human level, one has knowledge of knowledge and knowing of knowing (human only), and meaning of meaning. Knowledge and knowledge of knowledge are related contradictorially, that is, when one predominates (is actualized), the other is predominantly potentialized and vice versa, alternately and reciprocally. One can associate, with knowledge, Meaning 2 in order to say that knowledge, in contrast to Information, also has higher-order moral or esthetic aspects. By adding the step Meaning $2 \rightarrow$ Knowledge of Knowledge $\rightarrow$ Meaning 3, the feeling that Knowledge of Knowledge also has Meaning is captured, without their having to be identical. In other words, at this level, which is not about abstractions but real processes, Knowledge of knowledge and Meaning 3 are themselves in a contradictional interaction, in the sense that when one focuses on one aspect, the other is repressed (or potentialized).

LIR brings the "missing ingredient" of dynamic opposition or antagonism that reinforces this picture of meaning as significant information. It provides an explanation of how constraints as information in their physical manifestations can be causally effective when identified with the residual potentialities of informational processes. In this connection, using the same argument as that presented above, I can introduce the term constraining in addition to constraint, the participle giving a more accurate description as a process. Since LIR, unlike standard logics, is not topic-neutral, it can describe such informational interactions as logical processes. LIR proposes a new interpretation of the relation of meaning to modes of information, in that it considers that all modes of information involve a logical dynamics which ascends through levels of complexity and that that logical dynamics is the meaning corresponding to that level. At the cognitive level, information (as meaning) is organized into knowledge (or knowing) that subsumes normative and other values. 
In terms of this Special Issue, the relation of modes of information to meaning, the Deacon approach and Logic in Reality (LIR) thus accomplish two objectives: first of all, they ground and extend Logan's concept of the relativity of information, in that information is not only not an invariant quantity, but a process or set of processes of processes. Deacon's description of the evolution of intertwined thermo-, morpho- and teleodynamic levels further develops the concept of biotic or instructional information that results in the propagation of organization as pointed out by Kauffmann, Logan and their colleagues. In their concept of biotic information, biological or cognitive meaning is defined by interaction with the context (or environment) that interprets the information.

\section{Conclusions}

In the Overview of this paper, Section 1.3, I have stated a number of objectives, admittedly of different kinds, that I hope this paper has achieved. One goal is certainly to have opened up discussion of familiar scientific and philosophical concepts to a new logical perspective. Since the logic involved is of a completely different kind, metalogically, it cannot be judged by the standard criteria of what constitutes a logic. I therefore ask readers who are not yet willing to accept this new form of "non-classicality" to bracket their views of logic and judge my arguments and my conclusions in the following key areas, Deacon's absence and the existence of an informational paradigm, within their domain of discourse, for much further work.

\subsection{The Implications of Absence}

I have proposed an extension of Deacon's theory of incompleteness in nature two directions, both based on to the application of the LIR principle of dynamic opposition. LIR describes the logical structure and dynamics at the lowest levels of reality that underlie the simplest thermodynamic phenomena - the "materiality" of Logan-and provides further support for the significance of absence for processes at the highest cognitive levels of information and meaning, following the evolution of those processes through Deacon's levels of morpho- and teleodynamics.

As discussed in Section 5, Deacon has demonstrated that an inversion of perspective, focusing on what is absent rather than present, makes possible rethinking the frame of the natural sciences to give the necessary value to absential phenomena. As he states, there is more than what is actual. In the real world, there is, above all, what there could be, what is more or less really probable. In LIR terms, what is probable is not totally or absolutely absent, it is predominantly absent. At the level of semiotic intentionality and meaning, which is the one of real interest, what is present is not totally present, but is partly absent or unrealized, to use Deacon's term.

Deacon envisages in these complex phenomena, "our functions, meanings, experiences and values", a superposition of states of the present and absent that is similar to that of quantum mechanics and has been suggested by Nicolescu [33]. The difference is in the latter approach what goes into the equations (the orthomodular lattice to be more precise) are values of the (non-Kolmogorovian) probability that do not include the imaginary limiting values of zero and one that exist only for very simple processes at the thermodynamic (homeodynamic) level.

None of the dynamical properties associated with life and mind, such as purpose, representation and value, existed until the universe had evolved sufficiently to include complex molecules capable of 
entering into autogenic configurations. In Deacon's formulation, in the emergence of teleodynamics, nature finally discovered how to operate with the dynamical equivalent of zero. In the LIR picture, nature had discovered, from the "time" that anything physical existed, how to operate with the dynamical fact of absence and potentiality, always in a dynamical relation of conjunction with presence and actuality. In this modified conception, teleodynamic processes, those of the constraints affecting molecular dynamics, neuronal signal dynamics or sociological dynamics can be seen as embodying the probability values between zero and one, rather than being analogues of an abstract value of zero in the formal operations of matter-the dynamics of physical change.

Deacon has not minimized the further effort required to support his theory, and has admitted that his "proofs" in Incomplete Nature do not go beyond proofs of principle. In order to describe as rigorously and completely as possible the complex processes involving information, meaning and communication in human interactions, linguistic and other, without going outside the laws of physics, additional, again related principles must be applied to those processes. To repeat, LIR proposes, as physically grounded logical principles: (1) the constitutive role and causal efficacy of absence and potentiality; (2) the logical counteractive patterns of their evolution involving alternation between primarily actual and potential states of a process and its opposite; (3) the capacity of emergence of new entities from the interaction or opposition of the two.

In the conclusion of $\boldsymbol{I N}$, Deacon describes his view as "scientifically rigorous, but not simplistically materialistic" and that "despite its counterintuitive negative framing, the figure/background reversal of the way we conceive of living and mental causality promises to reinstate subjective experience as a legitimate participant in the web of physical causes and effects, and to ultimately reintroduce intentional phenomena back into the natural sciences.” This is the sole reason why I have considered it important to try to place on even firmer ground some of Deacon’s premises.

\subsection{The Informational Paradigm}

I have pointed elsewhere [7] to recent work in the philosophy of information by $\mathrm{Wu}$ as suggesting a radically new informational paradigm — a stance - in which both Deacon's and my conceptions are exemplified, coming from widely separated starting points in philosophy, biology and neuroscience and physics respectively. A new interpretation of Marijuan's "invisible hand" can now be proposed. Deacon and I are in agreement that information processes are involved in essentially all aspects of human existence, and both have proposed critical missing ingredients in prior explications of information. A new description of the nature and dynamics of information is provided as one example of the utility of combining the two approaches. As $\mathrm{Wu}$ has also stated, this is inevitably a transdisciplinary endeavor, since every major scientific and philosophical discipline is involved. It is impossible to proceed without some minimal understanding of the principles of physics, chemistry, cosmology, biology, neuroscience, psychology and social science on the one hand, and metaphysics, ontology, epistemology and logic on the other.

Deacon, the other authors I have pointed to and I have attempted to keep man at the center of our preoccupations. If the use of the concepts of classical, standard logic of absolute truth and falsity and the absolute independence of premises and conclusion contribute to the maintaining of Manichean views and policies in society, then anything that weakens the "stranglehold" of that logic on both 
formal and informal thought is to be welcomed. At the same time, as scientists, it is necessary to adhere to the most rigorous possible standards of argument, despite or perhaps because of our healthy measure of skepticism toward all received wisdom, both academic and folk. Other major contributions to this effort have been made, from the philosophical side, by Rafael Capurro and his followers in their concepts both of information ethics and of angeletics or messaging theory [34]. They start their discussion of messages and messaging from dissatisfaction with standard, limitative conceptions of information. It is possible to reconcile the former with the more adequately founded processual approaches to information outlined in this paper.

As Deacon has remarked, his work can supplement the combinatorial picture of Kauffmann and may allow us to be more truly "at home in the universe". The tentative consequences for the theory of information that emerge in part from this study are accordingly the following:

(i) Integrated consideration of physical and philosophical principles with dynamic principles derived from biology;

(ii) Acceptance of second- and third-order levels of information (information about information), without further regress;

(iii) Use of the concepts of dynamic constraints and absence to develop new understanding of the related, harder problems of sentience and the individual self;

(iv) Development of the characteristics of qualitative information involving human values, as a vital step in reintegrating issues of subjective value into the natural sciences;

(v) Further to the above, support for new, relevant conception of ethics for the information society.

The Principle of Dynamic Opposition of LIR, together with Deacon's theory, point toward what one reviewer has referred to as a "dynamic conversation" in the world that would be a major departure from the current still largely mechanical view of nature.

It is my hope that further discussion of Deacon's work, with specific reference to the articles in this Special Issue, will lead to further progress along these lines.

\section{Acknowledgements}

I would like to thank Terrence Deacon for our discussions of his work, and his encouragement to comment on it here and elsewhere. I have also much appreciated the helpful exchanges with Robert Logan and Robert Ulanowicz regarding key points in the formulation of Logic in Reality.

\section{References}

1. Marijuan, P.C. Information and life: Towards a biological understanding of informational phenomena. Triple-C 2004, 2, 6-19.

2. Hofkirchner, W. Twenty Questions about a Unified Theory of Information; Emergent Publications: Litchfield Park, AZ, USA, 2010.

3. Burgin, M. Theory of Information: Fundamentality, Diversity and Unification; World Scientific: New York, NY, USA, 2010.

4. Luhn, G. The Causal-compositional concept of information Part I. Elementary theory: From decompositional physics to compositional information. Information 2012, 3, 151-174. 
5. Deacon, T.W. What is missing from theories of information? In Information and the Nature of Reality: From Physics to Metaphysics; Davies, P., Gregersen, N.H., Eds.; Cambridge University Press: Cambridge, UK, 2010.

6. Deacon, T.W. Incomplete Nature: How Mind Evolved from Matter; W.W. Norton \& Co.: New York, NY, USA, 2012.

7. Wu, K.; Brenner, J.E. The informational stance. Logic and Philosophy. Log. Log. Philos. 2013, in press.

8. $\quad$ Brenner, J.E. Logic in Reality; Springer: Dordrecht, The Netherlands, 2008.

9. Machery, E. Why I stopped worrying about the definition of life... and why you should as well. Synthese 2012, 185, 145-164.

10. Ulanowicz, R.E. A Third Window: Natural Life beyond Newton and Darwin; Templeton Foundation Press: West Conshohocken, PA, USA, 2009.

11. Kauffman, S.; Logan, R.K.; Este, R.; Goebel, R.; Hobill, D.; Shmulevich, I. Propagating organization: An enquiry. Biol. Philos. 2007, 23, 27-45.

12. Brenner, J.E. Levels of abstraction. Levels of reality. Philos. Eng. Technol. 2012, 8, 201-222.

13. Brier, S. Cybersemiotics: Why Information is not Enough; University of Toronto Press: Toronto, Canada, 2008; p. 427.

14. Floridi, L. The logic of being informed. Log. Anal. 2006, 49, 433-460.

15. Zhong, X.-Y. On information science-An introduction to principles of information science. In Proceedings of the 4th International Conference on the Foundations of Information Science, Beijing, China, 21-24 August 2010.

16. Collier, J. Intrinsic information. In Information, Language, Cognition; Hanson, P., Ed.; University of British Columbia Press: Vancouver, Canada, 1990.

17. Brenner, J.E.; Burgin, M. Information as a natural and social operator. Inf. Theor. Appl. 2011, 18, 33-49.

18. Lupasco, S. Le Principe d'antagonisme et La Logique de L'énergie. Le Rocher: Paris, France, 1987.

19. Aerts, D.; D’Hondt, E.; Gabora, L. Why the logical disjunction in quantum logic is not classical. Found. Phys. 2000, 30, 1473-1480.

20. Lupasco, S. Qu'est-ce qu'une Structure? Christian Bourgois: Paris, France, 1967.

21. Varela, F.J. The creative circle: Sketches on the natural history of circularity. In The Invented Reality; Watzlavick, P., Ed.; Norton Publishing: New York, NY, USA, 1984.

22. Hofkirchner, W. How to achieve a unified theory of Information. Triple-C 2009, 7, 357-358.

23. Capurro, R. Towards an ontological foundation of information ethics. Ethics Inf. Technol. 2006, 8, 175-186.

24. Brenner, J.E. Information in reality: Logic and metaphysics. Triple-C 2011, 9, 332-341.

25. Deacon, T.W. Shannon-Boltzmann-Darwin: Redefining information (Part I). Cogn. Semiot. 2007, 1, 123-148.

26. Logan, R.K. What is information?: Why is it relativistic and what is its relationship to materiality, meaning and organization? Information 2012, 3, 68-91.

27. Ulanowicz, R.E. Towards quantifying a wider reality: Shannon exonerata. Information 2011, 2, 624-634.

28. Brenner, J.E. On representation in information theory. Information 2011, 2, 560-578. 
29. Lupasco, S. L'énergie et la Matière Vivante. Le Rocher: Monaco, 1987.

30. Varela, F.; Shear, J. First Person accounts: Why, what and how. In The View from Within; Varela, F., Shear, J., Eds.; Imprint Academic: Thorverton, UK, 1999; pp.1-14.

31. Brier, S. Cybersemiotics: An evolutionary world view going beyond entropy and information into the question of meaning. Entropy 2010 12, 1902-1920.

32. Heidegger, M. Being and Time; Oxford University Press: Oxford, UK, 1987.

33. Nicolescu, B. Nous, la Particule et le Monde. Editions du Rocher: Paris, France, 2002.

34. Capurro, R. Angeletics-A Message Theory. Messages and Messengers: Angeletics as an Approach to the Phenomenology of Communication; Capurro, R., Holgate, J., Eds.; Wilhelm Fink: Munich, Germany, 2011.

(C) 2012 by the authors; licensee MDPI, Basel, Switzerland. This article is an open access article distributed under the terms and conditions of the Creative Commons Attribution license (http://creativecommons.org/licenses/by/3.0/). 